\title{
Extended MSSM in supersymmetric SO(10) grand unification
}

\author{
Sibo Zheng* \\ Department of Physics, Chongqing University, Chongqing 401331, People's Republic of China
}

(Received 26 April 2018; published 16 August 2018)

\begin{abstract}
We apply the perturbative grand unification due to renormalization to distinguish TeV-scale relics of supersymmetric $\mathrm{SO}(10)$ scenarios. With rational theoretical constraints taken into account, we find that for the breaking pattern of either SU(5) or Pati-Salam, only extra matter 16 supermultiplet of $\mathrm{SO}(10)$ can appear at the TeV scale, apart from the MSSM spectrum.
\end{abstract}

DOI: 10.1103/PhysRevD.98.035028

\section{INTRODUCTION}

The discovery of the standard model (SM)-like Higgs [1,2] provides a new portal to $\mathrm{TeV}$-scale new physics at the $\mathrm{LHC}$ in the forthcoming years. Among other things, such new physics models may reveal the "nature" of the SM-like Higgs and offer a novel mechanism to stabilize divergence involving the SM Higgs. For those interesting scenarios in the literature, in this paper we are restricted to the idea of supersymmetry (SUSY). Specifically, we will utilize the grand unification (GUT) [3], which is one of the most beautiful features delivered by SUSY, to distinguish TeVscale relics of SUSY GUT models. For reviews on this subject, see e.g., $[4,5]$.

In the viewpoint of unification, the minimal supersymmetric standard model (MSSM) can be embedded into conventional $\mathrm{SU}(5)$ [6-8], $\mathrm{SO}(10)[9,10]$, or other GUT models with gauge groups of higher ranks. In light of our previous study on SU(5) [11], we will continue to explore the TeV-scale relics of SUSY SO(10) unification. Comparing with SU(5), the lowenergy effective theories of $\mathrm{SO}(10)$ are more complex. The first major reason is that there may be multiple intermediate scales between the weak and GUT scale. The second reason is that since a lot of higher-dimensional representations of $\mathrm{SO}(10)$ trivially satisfy gauge anomaly free condition, the constraint imposed by this condition is much weaker in $\mathrm{SO}(10)$. Earlier studies on low-energy effective theory which is consistent with perturbative SUSY SO(10) unification are based on specific motivations such as the Higgs mass [12] and neutrino physics [13-15].

Instead of particular phenomenological concerns, we will take a systematic analysis on the low-energy effective

\footnotetext{
*sibozheng.zju@gmail.com
}

Published by the American Physical Society under the terms of the Creative Commons Attribution 4.0 International license. Further distribution of this work must maintain attribution to the author(s) and the published article's title, journal citation, and DOI. Funded by SCOAP ${ }^{3}$. theory. In order to simplify the analysis on extra matter beyond the MSSM spectrum, we will explore $\mathrm{SO}(10)$ scenarios with the following theoretical features:

(i) The $\mathrm{SO}(10)$ unification is strictly perturbative.

(ii) In the chain of gauge symmetry breaking,

$$
\mathrm{SO}(10) \stackrel{H_{1}}{\longrightarrow} G_{1} \stackrel{H_{2}}{\longrightarrow} G_{2} \cdots \stackrel{H_{n}}{\longrightarrow} G_{\mathrm{SM}}
$$

where $G_{\mathrm{SM}}$ refers to the SM gauge group. When the Higgs component fields responsible for two nearby steps of gauge symmetry breaking can be contained in a single Higgs supermultiplet, these two Higgs supermultiplets will be identified as the same one. Otherwise, they differ from each other ${ }^{1}$.

(iii) In order to avoid dangerous mixings among Higgs vevs $\left\langle H_{i}\right\rangle$, all of $H_{i}$ are forbidden to directly couple to each other.

(iv) In order to avoid dangerous masses or mixing effects, neither the MSSM fields nor extra matters are allowed to directly couple to any Higgs supermultiplets $H_{i}$ in Eq. (1). ${ }^{2}$

The main reason for the last point is that the vacuum expectations (vevs) of $\left\langle H_{i}\right\rangle$ would result in large matter masses or large mixing effects if they were directly coupled to either MSSM fields or extra matters, which would lead to them playing no role at weak scale. For example, coupling a 54, which can break $\mathrm{SU}(5)$ to the $\mathrm{SM}$ gauge group, to MSSM Higgs $\mathbf{1 0}_{\mathrm{H}}$ through interaction $\mathbf{5 4} \times \mathbf{1 0}_{\mathrm{H}} \times \mathbf{1 0}_{\mathrm{H}}$, yields an unfavorable Higgsino mass for a large vev of SM singlet in $\langle\mathbf{5 4}\rangle$.

Theoretical constraints above have been partially imposed in the literature to our knowledge. However, they

\footnotetext{
${ }^{1}$ In this situation, the splitting between two nearby scales of gauge symmetry breaking is generally large. We will impose the relatively strong constraint that the two Higgs supermultiplets responsible for such large splitting are forbidden to directly couple to each other.

${ }^{2}$ There is a loophole. When the vev of the last Higgs $\left\langle H_{n}\right\rangle$ is of the order of the TeV scale, they are allowed to couple to $H_{n}$.
} 
have never been combined together to derive a systematic analysis on the low-energy effective theory. The paper is organized as follows. In Sec. II, we discuss the extra matter supermultiplets which are consistent with our starting points in two well known patterns of gauge symmetry breaking. In Sec. III, we examine the perturbative unification with these representations. Finally, we conclude in Sec. IV.

\section{REPRESENTATIONS}

According to our starting points, in this section we investigate the representation of extra matter which can directly couple to the SM Higgs $\mathbf{1 0}_{\mathbf{H}}$ in the following two patterns of gauge symmetry breaking,

$$
\begin{aligned}
& \mathrm{A}: \mathrm{SO}(10) \stackrel{H_{1}}{\longrightarrow} \mathrm{SU}(5) \stackrel{H_{2}}{\longrightarrow} G_{\mathrm{SM}}, \\
& \mathrm{B}: \mathrm{SO}(10) \stackrel{H_{1}}{\longrightarrow} \mathrm{SU}(4)_{c} \times \mathrm{SU}(2)_{L} \times \mathrm{SU}(2)_{R} \\
& \stackrel{H_{2}}{\longrightarrow} \mathrm{SU}(3)_{c} \times \mathrm{SU}(2)_{L} \times \mathrm{SU}(2)_{R} \times \mathrm{U}(1)_{B-L} \\
& \stackrel{H_{3}}{\longrightarrow} G_{\mathrm{SM}} .
\end{aligned}
$$

Pattern A [16-19] is a two-step breaking with $\mathrm{SU}(5)$ subgroup, and pattern B [20-22] is a three-step breaking referred to as the Pati-Salam model [21].

Note, in the MSSM the SM fermion matters are described by $\mathbf{1 6}_{i}$ of $\mathrm{SO}(10)$ with index $i=1-3$, and the SM Higgs is contained in the $\mathbf{1 0}_{\mathbf{H}}$ of $\mathrm{SO}(10)$. In particular, $\mathbf{1 6}_{i}$ contains three-generation right-hand neutrinos, whereas $\mathbf{1 0}_{\mathbf{H}}$ is composed of $\mathbf{5}_{\mathbf{H}}$ and $\overline{\mathbf{5}}_{\mathbf{H}}$ of $\mathrm{SU}(5)$ which contain the two Higgs doublets of MSSM and two color-triplets.

\section{A. SU(5)}

In this pattern of symmetry breaking, $H_{1}$ should contain an $\mathrm{SU}(5)$ singlet, there are two candidates $H_{1}=\{\mathbf{1 6}, \mathbf{1 2 6}\}$. The second Higgs $H_{2}$ should contain a 24 of SU(5), which corresponds to three potential choices $H_{2}=\{\mathbf{4 5}, \mathbf{5 4}, \mathbf{2 1 0}\}$. Since $H_{1} \neq H_{2}$, we take the rationale that the splitting between these two broken scales is large.

With potential assignments on $H_{1}$ and $H_{2}$ above, there are six sets of combinations. In each case, there may exist four types of dangerous gauge-invariant superpotentials which violate the last two starting points in Sec. I,

$$
\begin{aligned}
& H_{1} \times \mathcal{N} \times \mathcal{N} ; \\
& H_{2} \times \mathcal{N} \times \mathcal{N} ; \\
& H_{1} \times H_{2} \times \mathcal{N} ; \\
& H_{1} \times H_{1} \times H_{2}, H_{2} \times H_{2} \times H_{1},
\end{aligned}
$$

where MSSM matter field $\mathcal{N}=\left\{\mathbf{1 6}_{i}, \mathbf{1 0}_{\mathbf{H}}\right\}$. In Eq. (2), the first three types of superpotentials tender to seed Dirac or Majorana masses to MSSM matters or MSSM Higgs doublets; and the last type of gauge invariant superpotentials yields dangerous mixings between vevs of $H_{1}$ and $H_{2}$.

First, one finds a dangerous operator $16 \times \mathbf{1 6}_{i} \times \mathbf{1 0}_{\mathrm{H}}$ of the first type in Eq. (2) which disfavors the choice $H_{1}=\mathbf{1 6}$. Second, due to dangerous operator $\mathbf{1 2 6} \times \mathbf{2 1 0} \times \mathbf{1 0}_{\mathbf{H}}$ the set of $H_{1}=\mathbf{1 2 6}$ and $H_{2}=\mathbf{2 1 0}$ is also disfavored. Therefore, we are left with two combinations $H_{1}=\mathbf{1 2 6}$ and $H_{2}=\{\mathbf{4 5}, \mathbf{5 4}\}$. In the case $\left(H_{1}, H_{2}\right)=(\mathbf{1 2 6}, \mathbf{5 4})$, there exists a dangerous operator $126 \times \mathbf{1 2 6} \times \mathbf{5 4}$. In the last case, $\left(H_{1}, H_{2}\right)=(\mathbf{1 2 6}, \mathbf{4 5})$ denotes the new matter supermultiplets with $\mathcal{M}$, and we find that unsafe superpotentials exclude $\mathcal{M}=\{\mathbf{1 0}, \overline{\mathbf{1 6}}, 120,144,1 \overline{4} 4,210\}$, leaving us only two possibilities,

$$
16_{M} \times 16_{M} \times 10_{\mathrm{H}}, \quad 16_{M} \times 16_{i} \times 10_{\mathrm{H}} .
$$

Compared with breaking pattern A, there is another pattern of two-step breaking,

$$
S O(10) \stackrel{H_{1}}{\longrightarrow} G_{\mathrm{SM}} \times U(1) \stackrel{H_{2}}{\longrightarrow} G_{\mathrm{SM}} .
$$

In this case, the potential choices are $H_{1}=\{4 \mathbf{4 5}, 210\}$ and $H_{2}=\{\mathbf{1 6}, \mathbf{1 2 6}\}$. According to Eq. (2), dangerous operator $\mathbf{1 6} \times 1 \mathbf{1 6}_{i} \times 10_{\mathrm{H}}$ excludes the case $H_{2}=16$. Morevover, a dangerous operator $\mathbf{2 1 0} \times \mathbf{1 2 6} \times \mathbf{1 0}_{\mathbf{H}}$ excludes $H_{1}=\mathbf{2 1 0}$. Therefore, there is only a viable combination $\left(H_{1}, H_{2}\right)=$ $(45,126)$, in which case the extra matter $\mathcal{M}$ is similar to those of SU(5) subgroup.

\section{B. Pati-Salam}

In this pattern of symmetry breaking, $H_{1}$ should contain a singlet of $\mathrm{SU}(4)_{c} \times \mathrm{SU}(2)_{L} \times \mathrm{SU}(2)_{R}$, which has two choices $H_{1}=\{\mathbf{5 4}, \mathbf{2 1 0}\} . H_{2}$ should contain a singlet of $\mathrm{SU}(3)_{c}$ and $\mathrm{U}(1)_{B-L}$, which is a 15 of $\mathrm{SU}(4)_{C}$. There are two representations $H_{2}=\{\mathbf{4 5}, \mathbf{2 1 0}\}$ of $\mathrm{SO}(10)$ which include such a 15 . Finally, $H_{3}=\mathbf{1 6}$ offers the breaking of $\mathrm{SU}(2)_{R} \times \mathrm{U}(1)_{B-L} \rightarrow \mathrm{U}(1)_{Y}$.

Since 210 contains both a singlet of $\mathrm{SU}(4)_{c} \times \mathrm{SU}(2)_{L} \times$ $\mathrm{SU}(2)_{R}$ and a $\mathbf{1 5}$ of $\mathrm{SU}(4)_{c}$, according to the second starting point both $H_{1}$ and $H_{2}$ are identified as 210. In this case, the three-step breaking is approximately two-step. With $H_{1}=H_{2}=\mathbf{2 1 0}$, we are left with a single choice $\left(H_{1}, H_{2}, H_{3}\right)=(\mathbf{2 1 0}, \mathbf{2 1 0}, \mathbf{1 6})$, where similar to previous discussions about $\mathrm{SU}(5)$ there are four viable choices for extra matters,

$$
\begin{array}{ll}
16_{M} \times 16_{M} \times 10_{\mathbf{H}}, & 10_{M} \times 54_{M} \times 10_{\mathrm{H}}, \\
16_{M} \times 144_{M} \times 10_{\mathrm{H}}, & 144_{M} \times 1 \overline{4} 4_{M} \times 10_{\mathbf{H}} .
\end{array}
$$

Note, unlike in pattern A, extra matter supermultiplets in Eq. (4) are allowed to directly couple to $H_{3}$ because the broken scale of $\mathrm{SU}(2)_{R} \times \mathrm{U}(1)_{B-L}[21,23,24]$ can be close to the $\mathrm{TeV}$ scale (see, e.g., [25-30]). 


\section{PERTURBATIVE UNIFICATION}

With the theoretical constraints in the Introduction, we have clarified that a single or two $\mathbf{1 6}$ supermultiplets are allowed in pattern A, whereas two $16 \mathrm{~s}$, a 10 with 54, a 16 with 144, or a pair of vectorlike 144 may appear in the pattern B. Now, we examine whether any of them are consistent with the first constraint-perturbative unification.

We start with the one-loop renormalization group equations (RGEs) for SM gauge coupling constant,

$$
\frac{d}{d t} \alpha_{i}^{-1}=-\frac{b_{i}}{2 \pi}
$$

where RG scale $t=\ln \mu$ and coefficients $b_{i}=\left(b_{\mathrm{U}(1)_{Y}}\right.$, $\left.b_{\mathrm{SU}(2)_{L}}, b_{\mathrm{SU}(3)_{c}}\right)$ are determined by $[31,32]$

$$
b_{i}=-\left\{\frac{11}{3} C_{2}^{i}(G)-\frac{4}{3} \cdot \kappa \cdot T\left(r_{f_{i}}\right)-\frac{1}{6} T\left(r_{s_{i}}\right)\right\} .
$$

Here, $C_{2}(G)$ is the quadratic Casimir invariant, and $T(r)$ refers to dynkin index that depends on details of the representation [5].

\section{A. SU(5)}

In the case of $\mathrm{SU}(5)$ subgroup there are two intermediate scales $\Lambda_{\text {SUSY }}$ and $\Lambda_{5}$ between $M_{Z}$ and $\Lambda_{10}$, corresponding to SUSY and SU(5) breaking, respectively. The $b_{i}$ coefficients are given by ${ }^{3}$

$$
\begin{aligned}
& b_{i}= \begin{cases}(41 / 10,-19 / 6,-7), & \mu \in\left[M_{\mathrm{Z}}, \Lambda_{\mathrm{SUSY}}\right] \\
\left(33 / 5+\delta b_{1}(\mathbf{M}), 1+\delta b_{2 L}(\mathbf{M}),-3+\delta b_{3}(\mathbf{M})\right), & \mu \in\left[\Lambda_{\mathrm{SUSY}}, \Lambda_{5}\right]\end{cases} \\
& b_{5}=\delta b_{5}(\mathbf{M}) \text {, } \\
& \mu \in\left[\Lambda_{5}, \Lambda_{10}\right] \text {, }
\end{aligned}
$$

where $b_{5}$ denotes the $b$ coefficient of SU(5) subgroup, and $\delta b_{i}(\mathbf{M})$ refer to contributions to $b$ coefficients due to extra matter. In particular, $\delta b_{i}\left(\mathbf{1 6}_{M}\right)=(2,2,2)$ and $\delta b_{i}\left(\mathbf{1 6}_{M}+\mathbf{1 6}_{M}\right)=(4,4,4)$, respectively, in Eq. (7). Figure 1 shows the plots of RG running of SM gauge coupling constants according to Eq. (7). It reveals that for $\Lambda_{\mathrm{SUSY}}=1 \mathrm{TeV}$ the $\mathrm{SU}(5)$ unification occurs at $\Lambda_{5} \simeq 10^{16.3} \mathrm{GeV}$. Moreover, the $\mathrm{SO}(10)$ unification in both cases can occur at $\Lambda_{10}$ large than $10^{18} \mathrm{GeV}$. Comparing $\Lambda_{5}$ with $\Lambda_{10}$, one finds that there is indeed sufficient splitting between them, which verifies previous arguments.

\section{B. Pati-Salam}

In the case of Pati-Salam model there are two intermediate scales $\Lambda_{\mathrm{SUSY}}$ and $\Lambda_{\mathrm{R}}$ between $M_{Z}$ and $\Lambda_{10}$, which denotes SUSY and $S U(2)_{R} \times U(1)_{B-L}$ breaking scale, respectively. In this case, the coefficients $b_{i}$ are given by

$$
b_{i}= \begin{cases}(41 / 10,-19 / 6,-7), & \mu \in\left[M_{\mathrm{Z}}, \Lambda_{\mathrm{SUSY}}\right] \\ \left(33 / 5+\delta b_{1}(\mathbf{M}), 1+\delta b_{2}(\mathbf{M}),-3+\delta b_{3}(\mathbf{M})\right), & \mu \in\left[\Lambda_{\mathrm{SUSY}}, \Lambda_{\mathrm{R}}\right]\end{cases}
$$

for the RG scale between $M_{Z}$ and $\Lambda_{R}$, and

$$
b_{i}=\left(6+2+\delta b_{B-L}(\mathbf{M}), 0+2+\delta b_{2 L}(\mathbf{M}), 0+2+\delta b_{2 R}(\mathbf{M}),-3+2+\delta b_{3}(\mathbf{M})\right), \quad \mu \in\left[\Lambda_{R}, \Lambda_{10}\right]
$$

for the RG scale between $\Lambda_{\mathrm{R}}$ and $\Lambda_{10}$. Above the RG scale $\Lambda_{R}$, MSSM matters and Higgs field $H_{3}=\mathbf{1 6}$ contributes to $\delta b_{i}=(6,0,0,-3)$ and $\delta b_{i}=(2,2,2,2)$ in Eq. (9), respectively. Regardless of what extra matter appears above $\Lambda_{\text {SUSY }}$ and what kind of Higgs $H_{1,2,3}$ above $\Lambda_{R}, \operatorname{SO}(10)$

\footnotetext{
${ }^{3}$ In order to eliminate the triplet fields in $\mathbf{1 0}_{\mathbf{H}}$ some additional matter representations may be required as well. Nevertheless, they are usually decoupled below the scale $\Lambda_{5}$, which don't affect the RG analysis between $M_{Z}$ and $\Lambda_{5}$.
}

unification at the one-loop level yields $\ln \left(\Lambda_{10} / \Lambda_{R}\right) \simeq 2.53$ [33,34], which uniquely determines $\Lambda_{\mathrm{R}}$ once the content of extra matters is identified.

Take a pair of $\mathbf{1 6}_{M}$ e.g., they contribute to $\delta b_{i}(\mathbf{M})=$ $(4,4,4)$ and $\delta b_{i}(\mathbf{M})=(4,4,4,4)$ in Eq. (8) and Eq. (9), respectively, which gives rise to $\Lambda_{\mathrm{R}} \simeq 10^{15.5} \mathrm{GeV}$, unified gauge coupling $\alpha^{-1} \simeq 5.45$, and $\Lambda_{10} \simeq 10^{16.7} \mathrm{GeV}$. Figure 2 shows the RG running of SM gauge coupling constant, which offers us perturbative $\mathrm{SO}(10)$ unification. Note that the RG running of the $S U(2)_{R}$ gauge coupling 


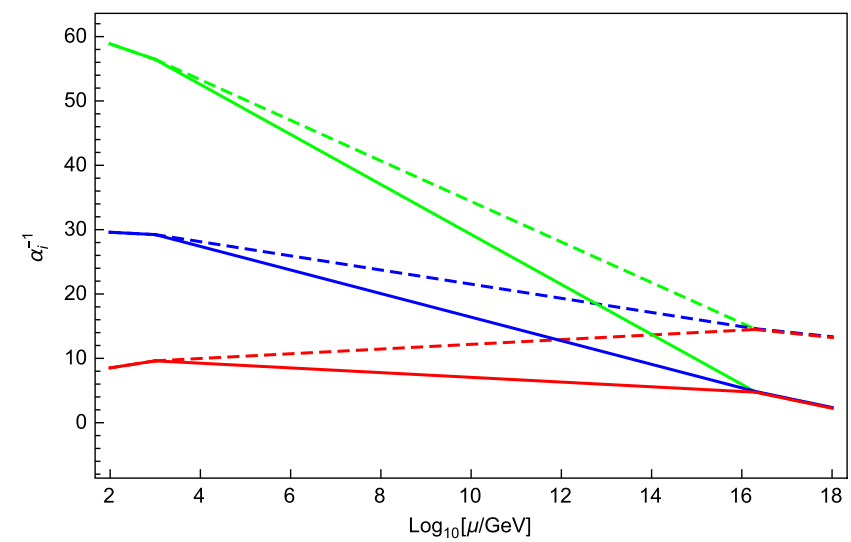

FIG. 1. One-loop RGEs for SM gauge coupling $\alpha_{1}^{-1}$ (green), $\alpha_{2}^{-1}$ (blue) and $\alpha_{3}^{-1}$ (red) for extra matter $\mathbf{1 6}_{M}$ (dashed) and $\mathbf{1 6}_{M}+\mathbf{1 6}_{M}$ (solid), respectively. Here SUSY breaking scale $\Lambda_{\mathrm{SUSY}}=1 \mathrm{TeV}$.

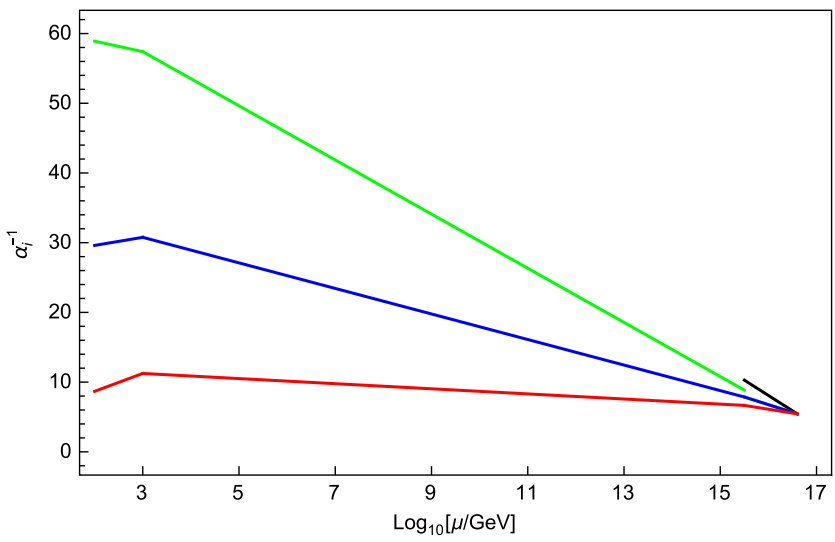

FIG. 2. One-loop RGEs for SM gauge coupling $\alpha_{1}^{-1}$ (green), $\alpha_{2 L}^{-1}$ (blue), $\alpha_{3}^{-1}$ (red) and $\alpha_{\mathrm{B}-\mathrm{L}}^{-1}$ (black) for extra matter $\mathbf{1 6}_{M}+\mathbf{1 6}_{M}$. For $\Lambda_{\text {SUSY }}=1 \mathrm{TeV}$, one obtains $\Lambda_{\mathrm{R}} \simeq 10^{15.5} \mathrm{GeV}$ and $\Lambda_{10} \simeq 10^{16.7} \mathrm{GeV}$. The $\mathrm{RG}$ running of $\mathrm{SU}(2)_{R}$ gauge coupling constant between $\Lambda_{R}$ and $\Lambda_{10}$ coincides with that of $\mathrm{SU}(2)_{L}$.

constant between $\Lambda_{R}$ and $\Lambda_{10}$ coincides with that of $S U(2)_{L}$, and as required $\alpha_{Y}^{-1}$ is equal to $\frac{3}{5} \alpha_{2 R}^{-1}+\frac{2}{5} \alpha_{B-L}^{-1}$ at RG scale $\Lambda_{R}$.
We repeat the analysis for other choices on extra matters in Eq. (4). We find that, in these cases, $b$ coefficients such as $\delta b_{i}\left(\mathbf{1 0}_{M}+\mathbf{5 4}_{M}\right)=(11.5,11.5,11.5,11.5)$ are always too large to support the idea of perturbative unification.

\section{CONCLUSION}

In the forthcoming years, we will enter into a new era of precise Higgs physics, which means that studying new physics through the Higgs portal will become very interesting. In this paper, we have utilized perturbative unification due to renormalization to explore the low energy effective theory of SUSY $\mathrm{SO}(10)$ scenarios. With the rational theoretical constraints taken into account, we find that for the breaking pattern of either $\mathrm{SU}(5)$ or Pati-Salam only 16 supermultiplet can appear at the $\mathrm{TeV}$ scale apart from the MSSM spectrum.

The quarks or leptons in the $\mathbf{1 6}$ supermultiplet(s) can be either chiral or vectorlike. Note, the vectorlike fermion mass requires addition of SM singlet (with vev of order $\mathrm{TeV}$ ) which does not affect our discussions. While the chiral case has been excluded, the vectorlike quarks or leptons are smoking guns in these SUSY SO(10) scenarios. Moreover, the neutral fermions of singlet or doublets of the $\mathbf{1 6}$ supermultiplet can serve as dark matter totally, or partially with the neutralinos of the MSSM.

\section{ACKNOWLEDGMENTS}

The author is grateful to Dr. J. Zhang for discussions. The author thanks the referee for helpful comments on the manuscript and for reminding us of alternative pattern in the case of $\mathrm{SU}(5)$. This work is supported, in part, by the National Natural Science Foundation of China under Grant No. 11775039 and the Fundamental Research Funds for the Central Universities with Project No. cqu2017hbrc1B05 at Chongqing University.
[1] G. Aad et al. (ATLAS Collaboration), Phys. Lett. B 716, 1 (2012).

[2] S. Chatrchyan et al. (CMS Collaboration), Phys. Lett. B 716, 30 (2012).

[3] H. Georgi and S. L. Glashow, Phys. Rev. Lett. 32, 438 (1974).

[4] P. Langacker, Phys. Rep. 72, 185 (1981).

[5] R. Slansky, Phys. Rep. 79, 1 (1981).
[6] S. Dimopoulos and H. Georgi, Nucl. Phys. B193, 150 (1981).

[7] N. Sakai, Z. Phys. C 11, 153 (1981).

[8] S. Dimopoulos, S. Raby, and F. Wilczek, Phys. Rev. D 24, 1681 (1981).

[9] H. Georgi, in Particles and Fields, edited by C. E. Carlson (AIP, New York, 1975), p. 575.

[10] H. Fritzsch and P. Minkowski, Ann. Phys. (N.Y.) 93, 193 (1975). 
[11] S. Zheng, Eur. Phys. J. C 77, 588 (2017).

[12] K. S. Babu, I. Gogoladze, M. U. Rehman, and Q. Shafi, Phys. Rev. D 78, 055017 (2008).

[13] K. S. Babu and R. N. Mohapatra, Phys. Rev. Lett. 70, 2845 (1993).

[14] H. S. Goh, R. N. Mohapatra, and S. Nasri, Phys. Rev. D 70, 075022 (2004).

[15] P. S. B. Dev and R. N. Mohapatra, Phys. Rev. D 81, 013001 (2010).

[16] H. Georgi and D. V. Nanopoulos, Phys. Lett. 82B, 392 (1979).

[17] H. Georgi and D. V. Nanopoulos, Nucl. Phys. B155, 52 (1979).

[18] H. Georgi and D. V. Nanopoulos, Nucl. Phys. B159, 16 (1979).

[19] S. Rajpoot, Phys. Rev. D 22, 2244 (1980).

[20] M. S. Chanowitz, J. Ellis, and M. K. Galllard, Nucl. Phys. B128, 506 (1977).

[21] J. C. Pati and A. Salam, Phys. Rev. D 10, 275 (1974); 11, 703(E) (1975).

[22] A. Masiero, Phys. Lett. B 93, 295 (1980).
[23] R. N. Mohapatra and J. C. Pati, Phys. Rev. D 11, 2558 (1975).

[24] G. Senjanovic and R. N. Mohapatra, Phys. Rev. D 12, 1502 (1975).

[25] G. Beall, M. Bander, and A. Soni, Phys. Rev. Lett. 48, 848 (1982).

[26] G. Ecker, W. Grimus, and H. Neufeld, Nucl. Phys. B229, 421 (1983).

[27] P. Langacker and S. U. Sankar, Phys. Rev. D 40, 1569 (1989).

[28] Y. Zhang, H. An, X. Ji, and R. N. Mohapatra, Nucl. Phys. B802, 247 (2008).

[29] A. Maiezza, M. Nemevsek, F. Nesti, and G. Senjanovic, Phys. Rev. D 82, 055022 (2010).

[30] S. Bertolini, A. Maiezza, and F. Nesti, Phys. Rev. D 89, 095028 (2014).

[31] M. E. Machacek and M. T. Vaughn, Nucl. Phys. B236, 221 (1984).

[32] M. E. Machacek and M. T. Vaughn, Nucl. Phys. B249, 70 (1985).

[33] M. Bastero-Gil and B. Brahmachari, Nucl. Phys. B482, 39 (1996).

[34] R. N. Mohapatra, arXiv:hep-ph/9911272. 